\title{
Nutritional stress of adult female tsetse flies (Diptera: Glossinidae) affects the susceptibility of their offspring to trypanosomal infections
}

\author{
K. Akoda ${ }^{\text {b }}$, J. Van Den Abbeele ${ }^{\text {b }}$, T. Marcotty ${ }^{a}$, R. De Deken ${ }^{a}$, I. Sidibe ${ }^{c}$, P. Van den Bossche ${ }^{\mathrm{a}, \mathrm{d}, *}$ \\ a Department of Animal Health, Institute of Tropical Medicine, Antwerp, Belgium \\ ${ }^{\mathrm{b}}$ Department of Parasitology, Institute of Tropical Medicine, Antwerp, Belgium \\ c Centre International de Recherche-Développement sur l'Elevage en zone Subhumide, Bobo-Dioulasso, Burkina Faso \\ d Department of Veterinary Tropical Diseases, University of Pretoria, Onderstepoort, South Africa
}

\section{A R T I C L E I N F O}

\section{Article history:}

Received 16 June 2008

Received in revised form 5 May 2009

Accepted 6 May 2009

Available online xxx

\section{Keywords:}

Glossina morsitans

Trypanosoma congolense

Trypanosoma brucei brucei

Starvation

Offspring

Vectorial capacity

\begin{abstract}
A B S T R A C T
The epidemiology of tsetse-transmitted trypanosomiasis depends, among other factors, on the proportion of infected flies in a tsetse population. A wide range of intrinsic and extrinsic factors seem to determine the ability of a tsetse fly to become infected and to transmit the parasite. In this paper, we investigated the effect of nutritional stress of reproducing female Glossina morsitans morsitans on the susceptibility of their offspring to trypanosomal infections. Adult female flies that were nutritionally stressed by feeding only once a week, produced pupae with a significant lower weight and offspring with a significant lower fat content as well as a lower baseline immune peptide gene expression. Moreover, infection experiments showed that the emerging teneral flies were significantly more susceptible to a Trypanosoma congolense or Trypanosoma brucei brucei infection than flies emerging from non-starved adult females. These findings suggest that in the field, substantial nutritional stress of adult tsetse flies, as is often experienced during the hot dry season, can increase significantly the vectorial capacity of the emerging teneral flies and thus result in an increased infection rate of the tsetse population.
\end{abstract}

(c) 2009 Elsevier B.V. All rights reserved.

\section{Introduction}

In Africa, tsetse-transmitted trypanosomiasis poses a serious threat to animals and humans. About 10 million $\mathrm{km}^{2}$ of sub-Saharan Africa, extending over 37 countries, are infected by tsetse flies. The epidemiology of this important disease is determined largely by the proportion of infected tsetse flies. A range of intrinsic and extrinsic factors among which environmental conditions, have been identified to affect the tsetse's susceptibility to trypanosomal infections and, thus affect the overall infection rate of the tsetse population (Leak, 1998; Aksoy et al., 2003; Macleod et al., 2007). For example, infection rates in tsetse flies are positively correlated with ambient temperature to which puparia or adult flies are exposed (Taylor, 1932; Burtt, 1946; Fairbairn and Watson, 1955; Ndegwa et al., 1992). Moreover, high ambient temperatures shorten the duration of the development cycle within tsetse (Fairbairn and Culwick, 1950). The underlying mechanisms for the observed effect of ambient temperature on the susceptibility to infection are not well understood. The

\footnotetext{
* Corresponding author at: Prince Leopold Institute of Tropical Medicine, Department of Animal Health, Nationalestraat 155, B-2000 Antwerp, Belgium. Tel.: +32 3247 6396; fax: +32 32476268 .

E-mail address: pvdbossche@itg.be (P. Van den Bossche).
}

nutritional status of the tsetse fly at the time of the infective bloodmeal also affects its susceptibility to infections with Trypanosoma congolense or Trypanosoma brucei brucei. Indeed, a period of starvation (4 days for teneral flies and 7 days for adult flies) lowers the developmental barrier for a trypanosome infection, especially at the midgut level of the fly (Kubi et al., 2006). Here, it has been hypothesized that the reduction in the fat body reserve as a result of starvation reduces the fly's ability to mount a competent immune response against the invading trypanosomes. It has been suggested that the tsetse's innate immune response affects the establishment and maturation of trypanosomes in the vector (Hao et al., 2001; Boulanger et al., 2002; Lehane et al., 2004; Attardo et al., 2006). This immune response is determined by a range of antimicrobial peptides among which attacin, defensin, cecropin and diptericin that are mainly synthesized in the fat body in response to a 'foreign' micro-organism. Hence, all factors that reduce the fat body level of a teneral or adult fly may result in concomitant changes in the fly's vector competence. In this paper, we investigated the effect of nutritional stress of reproducing tsetse females, on the susceptibility of their offspring to trypanosomal infection. Results of this study may explain how changes in the tsetse's environment, that cause considerable stress, may have repercussions on the epidemiology of tsetse-transmitted human or animal trypanosomiasis.

0001-706X/\$ - see front matter (c) 2009 Elsevier B.V. All rights reserved. doi:10.1016/j.actatropica.2009.05.005 


\section{Materials and methods}

\subsection{Tsetse flies}

Tsetse flies Glossina morsitans morsitans Westwood from the colony maintained at the Institute of Tropical Medicine (Antwerp, Belgium) were used in the experiments. The origin of this tsetse colony and rearing conditions were described by Elsen et al. (1993). Flies from this colony were fed 4 times a week on healthy rabbits (=non-starved colony). In addition to the main colony, a second colony of 2220 adult reproducing female flies was established and nutritionally stressed by feeding the flies only once a week on healthy rabbits (=starved colony) for 9 weeks. Pupae produced by the female flies of both colonies were collected daily and weighed.

\subsection{Trypanosome infection of tsetse flies}

The clonal strains of $T$. congolense IL 1180 (Geigy and Kauffman, 1973) and T. brucei brucei AntAR1 (Le Ray et al., 1977) were used to infect the experimental flies. Series of 30 male flies (less than $32 \mathrm{~h}$ old) that emerged from pupae produced by females of the starved or non-starved colony were given a single bloodmeal on trypanosome infected anesthetized mice (NMRI). The mice were infected with either $T$. congolense or $T$. b. brucei showing a parasitaemia of $10^{6.9}$ or $10^{8.1}$ trypanosomes per ml, respectively. Mice were anesthetized with $0.05 \mathrm{ml}$ of a $8 / 3(\mathrm{v} / \mathrm{v})$ mixture of Anesketin ${ }^{\circledR}$ (diluted to $5 \%$ ketamine in physiological water) and Rompun ${ }^{\circledR}$. Only fully engorged flies were retained and fed afterwards on healthy rabbits three times per week. To avoid re-infection of the flies, these rabbits were replaced at weekly intervals.

\subsection{Tsetse infection rate}

Twenty-one days (for infections with $T$. congolense) or 30 days (for infections with T. b. brucei) after the infective bloodmeal, all surviving flies were dissected using the method described by Llyod and Johnson, 1924. Microscopical examination of the midgut and mouthparts or midgut and salivary glands were conducted to determine the presence of $T$. congolense (infection of midgut and mouthparts) or T. b. brucei (infection of midgut and salivary glands), respectively.

The proportion of immature (procyclic) infections was calculated as the proportion of dissected flies that had a trypanosomal infection in the midgut whereas the proportion of mature (metacyclic) infections was calculated as the proportion of dissected flies infected in midgut and mouthparts (T. congolense) or midgut and salivary glands (T. b. brucei). The maturation rate was calculated as the proportion of midgut infection that developed into a mature infection.

\subsection{Determination of the fat content in the offspring}

Thirty (30) male flies that emerged from pupae of the starved or non-starved colony were killed immediately after emergence, their legs and wings were removed and the carcasses were dried at $80^{\circ} \mathrm{C}$ to constant weight. Afterwards the dry weight of pools of three flies was determined. Fat body was extracted through chloroform extraction of lipids for three days with daily changes of chloroform (Langley et al., 1990). After fat extraction and drying to constant weight, the residual dry weight of the pools of three flies was determined. The fat content of the flies was calculated as the difference between the dry weight and the residual dry weight. Weighing was done using a Sartorius ${ }^{\circledR}$ (GMBH, A120S) analytical balance $\left(10^{-4} \mathrm{~g}\right)$.

\subsection{Immune peptide gene expression levels in the freshly emerged flies}

To compare the baseline immune peptide gene expression level of male flies derived from pupae of the starved or non-starved adult females, total RNA was extracted from the whole abdomens of respectively 24 and 20 male flies. RNA extraction was conducted in $1 \mathrm{ml}$ Tripure ${ }^{\circledR}$ reagent (Roche) per sample following the manufacturer's instructions. Afterwards, the mRNA in $400 \mathrm{ng}$ of a total RNA extract was reverse transcribed using Oligo(dT) ${ }_{15}$ primer (100 pmol) (Promega), dNTP-mix (10 mM each) and Transcriptor Reverse Transcriptase (10 units) (Roche). Quantitative real-time PCR was performed in a Bio-Rad iCycler iQ with Bio-Rad iQ SYBR green Supermix. PCR conditions comprised an initial 10 min polymerase activation at $95^{\circ} \mathrm{C}$ followed by 35 cycles each consisting of a denaturation step at $95^{\circ} \mathrm{C} / 15 \mathrm{~s}$ and an annealing/elongation step at $60^{\circ} \mathrm{C}$. Used primers were: attacinFW: $5^{\prime}$-TTTTCACAGTCGCACCCATT-3' and attacinREV: 5'-AAACGCCTCCTGTCAAATCC-3', defensinFW: 5'-TAGTTTTGGCTTTTCTTACAC-3' and defensinREV: $5^{\prime}$ CGACTACAGTATCCGCTCTTT-3', and cecropinFW: 5'-ATACTCGCTCTTTCAGTCAG-3' and cecropinREV: 5'-CTCTAACAGTAGCGGCAACA3'; actinFW: 5'-CGCTTCTGGTCGTACTACT-3' and actinREV: 5'-CCGGACATCACAATGTTGG-3', tubulinFW: 5'-GATGGTCAAGTGCGATCCT$3^{\prime}$ and tubulinREV: 5'-TGAGAACTCGCCTTCTTCC- $3^{\prime}$. Three replicates were performed for each sample and threshold cycles $(C t)$ were recorded and used to calculate gene expression levels.

\subsection{Statistical data analysis}

All statistical analyses were carried out in Stata software version 9.2 (StataCorp, 2006). A linear regression was used to analyse the loss of weight in individual flies. The difference in weight was used as the response and fly groups (flies emerging from pupae produced by females of the starved and non-starved colony) as discrete explanatory variables. The distribution of the residuals and the heteroskedasticity of the variance were verified $(p>0.1)$.

The proportions of infected flies in different experimental series were compared using a robust logistic regression. Cluster effects resulting from flies infected by feeding on the same mouse and maintained in the same cage were taken into account in the model.

The effect of adult female starvation on the expression levels of attacin, defensin and cecropin in emerging teneral male flies were analysed using a robust linear model. The response variables were the logarithm of the normalized number of cycles needed for the immune peptides specific cDNA to reach the threshold in real-time PCR. The normalization used was a modification of that proposed by Vandesompele et al. (2002):

response $=\ln \left[\left(\mathrm{PCR}_{\mathrm{pep}}\right)^{C t_{\mathrm{pep}}}\right]-\frac{\ln \left[\left(\mathrm{PCR}_{\mathrm{act}}\right)^{C t_{\mathrm{act}}}\right]+\ln \left[\left(\mathrm{PCR}_{\mathrm{tub}}\right)^{C t_{\mathrm{tub}}}\right]}{2}$

with:

- $\mathrm{PCR}_{\text {pep/act/tub }}=\mathrm{PCR}$ efficiency of immune peptides, actin and tubulin, respectively

- $C t_{\text {pep }}=$ number of cycles required for immune peptides genes to reach the threshold in each sample repetition

- $C t_{\text {act/tub }}=$ three repetition average number of cycles required for actin and tubulin genes, respectively to reach the threshold in each sample

Clustering effects resulting from repeated measures of the same samples were taken into account in the model. Discrete explanatory variables consisted of the different experimental groups created in each experiment. For each statistical analysis, differences were considered significant when $p<0.05$. 


\section{Results}

During the study period, the starved colony produced 0.07-0.15 pupae per female per week whereas the non-starved colony produced about 1.0 pupae per female per week. Many abortions were observed in the starved colony. The pupal mean weight ranged between 20 and $22 \mathrm{mg}$ in the starved colony while the pupal mean weight in the non-starved colony was about $26 \mathrm{mg}$. The pupal emergence rate was $89 \%$ in the starved colony and $97 \%$ in the non-starved female colony.

\subsection{Nutritional stress and expression of immune peptide genes of the emerging flies}

Male flies emerging from pupae produced by the non-starved females had an almost 2-fold higher fat body content than teneral males emerging from pupae produced by the starved females $(2.5 \pm 0.1 \mathrm{mg}$ compared to $1.4 \pm 0.4 \mathrm{mg}, p<0.0001)$. The normalized gene expression levels of the immune peptides attacin, defensin and cecropin were respectively 1.5, 1.8 and 2.0 times higher in teneral male flies from non-starved adult females (Fig. 1). None of these differences are, however, statistically significant.

\subsection{Trypanosome infection rates}

The proportion of teneral males feeding on the infected mice was $74.8 \%$ and $65.6 \%$ for tenerals emerging from pupae produced by the starved and the non-starved females, respectively. Mortality rates of flies after the infective feed were $7.1 \%$ and $1.5 \%$ for males emerging from pupae produced by starved and non-starved females, respectively.

The proportion of flies from the starved colony that developed a mature infection with $T$. congolense (Table 1 ) or T. b. brucei (Table 2) was significantly higher compared to flies from the non-starved colony $(p<0.001)$. In $T$. congolense-infected flies, the proportion

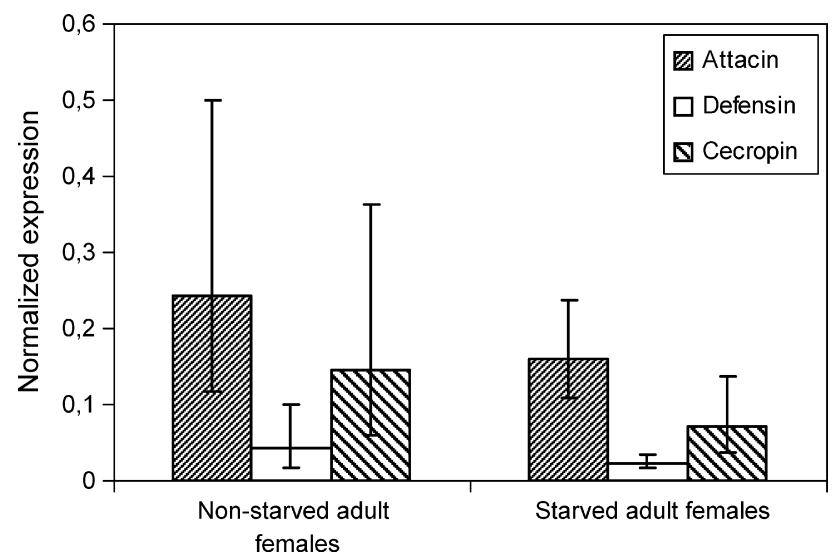

Fig. 1. Normalized expression levels of attacin, defensin and cecropin in male teneral G. m. morsitans emerging from non-starved or starved adult female flies and their 95\% confidence intervals. Expression level of each immune gene was normalized against the value of the housekeeping genes actin and tubulin.

of male flies that developed a midgut infection was significantly higher in the flies emerging from the starved colony compared to flies from the non-starved colony ( 0.44 versus $0.27, p=0.0001)$. The maturation rate of the midgut infections was about $100 \%$ in both experimental groups. For infections with $T$. $b$. brucei, the proportion of flies that developed a midgut infection did not differ significantly between the experimental groups $(p=0.067)$ but the maturation rate was significantly higher in males emerged from pupae produced by females of the starved colony ( 0.55 versus 0.35 ; $p=0.011$ ) (Table 2 ).

\section{Discussion}

The starvation induced in the adult female tsetse flies substantially increased their mortality rate and reduced their reproductive

Table 1

Proportion of male teneral G. m. morsitans emerging from starved or non-starved females and infected with T. congolense IL1180.

\begin{tabular}{|c|c|c|c|c|c|}
\hline \multirow[t]{2}{*}{ Experimental group } & \multirow[t]{2}{*}{ Batch of flies } & \multirow[t]{2}{*}{ Number dissected } & \multicolumn{2}{|c|}{ Proportion of flies infected in } & \multirow[t]{2}{*}{ Maturation rate } \\
\hline & & & Midgut (immature) & Mouthparts (mature) & \\
\hline \multirow[t]{6}{*}{ Male teneral flies emerging from starved adult females } & 1 & 28 & $0.50(14 / 28)$ & $0.50(14 / 28)$ & 1.00 \\
\hline & 2 & 17 & $0.41(7 / 17)$ & $041(7 / 17)$ & 1.00 \\
\hline & 3 & 25 & $0.40(10 / 25)$ & $0.40(10 / 25)$ & 1.00 \\
\hline & 4 & 27 & $0.41(11 / 27)$ & $0.41(11 / 27)$ & 1.00 \\
\hline & 5 & 25 & $0.48(12 / 25)$ & $0.44(11 / 25)$ & 0.92 \\
\hline & 6 & 17 & $0.41(7 / 17)$ & $0.41(7 / 17)$ & 1.00 \\
\hline \multirow[t]{6}{*}{ Male teneral flies emerging from non-starved adult females } & $1^{\prime}$ & 15 & $0.20(3 / 15)$ & $0.20(3 / 15)$ & 1.00 \\
\hline & $2^{\prime}$ & 22 & $0.32(7 / 22)$ & $0.32(7 / 22)$ & 1.00 \\
\hline & $3^{\prime}$ & 40 & $0.30(12 / 40)$ & $0.30(12 / 40)$ & 1.00 \\
\hline & $4^{\prime}$ & 46 & $0.26(12 / 46)$ & $0.26(12 / 46)$ & 1.00 \\
\hline & $5^{\prime}$ & 28 & $0.25(7 / 28)$ & $0.25(7 / 28)$ & 1.00 \\
\hline & $6^{\prime}$ & 31 & $0.29(9 / 31)$ & $0.29(9 / 31)$ & 1.00 \\
\hline
\end{tabular}

Table 2

Proportion of male teneral G. m. morsitans emerging from starved or non-starved females and infected with T. b. brucei AnTAR 1.

\begin{tabular}{|c|c|c|c|c|c|}
\hline \multirow[t]{2}{*}{ Experimental group } & \multirow[t]{2}{*}{ Batch of flies } & \multirow[t]{2}{*}{ Number dissected } & \multicolumn{2}{|c|}{ Proportion of flies infected in } & \multirow[t]{2}{*}{ Maturation rate } \\
\hline & & & Midgut (immature) & Salivary glands (mature) & \\
\hline \multirow[t]{5}{*}{ Male teneral flies emerging from starved adult females } & 1 & 16 & $0.56(9 / 16)$ & $0.25(4 / 16)$ & $0.44(4 / 9)$ \\
\hline & 2 & 20 & $0.65(13 / 20)$ & $0.35(7 / 20)$ & $0.54(7 / 13)$ \\
\hline & 3 & 18 & $0.61(11 / 18)$ & $0.39(7 / 18)$ & $0.64(7 / 11)$ \\
\hline & 4 & 15 & $0.53(8 / 15)$ & $0.33(5 / 15)$ & $0.63(5 / 8)$ \\
\hline & 5 & 28 & $0.64(18 / 28)$ & $0.33(9 / 28)$ & $0.50(9 / 18)$ \\
\hline \multirow[t]{5}{*}{ Male teneral flies emerging from non-starved adult females } & $1^{\prime}$ & 20 & $0.55(11 / 20)$ & $0.10(2 / 20)$ & $0.18(2 / 11)$ \\
\hline & $2^{\prime}$ & 26 & $0.58(15 / 26)$ & $0.15(4 / 26)$ & $0.27(4 / 15)$ \\
\hline & $3^{\prime}$ & 25 & $0.40(10 / 25)$ & $0.24(6 / 25)$ & $0.60(6 / 10)$ \\
\hline & $4^{\prime}$ & 27 & $0.52(14 / 27)$ & $0.22(6 / 27)$ & $0.43(6 / 14)$ \\
\hline & $5^{\prime}$ & 43 & $0.58(25 / 43)$ & $0.16(7 / 43)$ & $0.28(7 / 25)$ \\
\hline
\end{tabular}


performance. Moreover, the mortality rate of the offspring of those starved females was substantially higher compared to that of offspring of the non-starved females. This suggests a reduced viability of male tsetse flies produced by female flies submitted to this level of nutritional stress. This is not surprising considering the tsetse's adenotrophic viviparity and the importance of the uterine milk produced by the mother fly for the development of the three larval stages in its uterus (Denlinger and Ma, 1974). Consequently, the condition of the mother fly has direct repercussions for its offspring.

Environmental conditions vary seasonally and have a significant effect on the fat content and size of adult tsetse flies (Glasgow and Bursell, 1960; Van den Bossche and Hargrove, 1999) as well as on the body size and survival of their offspring (Jackson, 1952; Phelps and Clarke, 1974; Dransfield et al., 1989). Based on the performance parameters of the starved colony, the nutritional stress induced in this colony seems to mimic the seasonal stress experienced by field populations of tsetse flies. Especially the reduced fat level of the emerging teneral male flies may be attributed directly to the nutritional stress the female flies were subjected to. In accordance with observations made by Kubi et al. (2006), the mature trypanosomal infection rate of teneral males with low fat levels (emerging from pupae produced by females from the starved colony) was significantly higher than the infection rates of males with higher fat levels (emerging from pupae produced by the non-starved colony). This increased susceptibility to trypanosomal infections could be attributed partially to a reduced baseline immune status of the offspring as a result of the nutritional stress experienced by the parent fly. Such hypothesis is supported by Koella and Sörensen's (2002) observations that sufficient and good quality food is essential for good immune defence in insects. Similarly, Siva-Jothy and Thompson (2002) concluded that a reduction in resource availability could decrease the immune function of invertebrates. In our study, the impact of starvation on the immune system was only examined for immune peptides that are mainly produced systemically by the fat body and used by the tsetse's defence mechanisms against trypanosome infection (Hao et al., 2001; Boulanger et al., 2002). The fact that the expression of these immune peptides genes was reduced, but not significantly so, could be due to the way in which this expression was measured. Indeed, measurement of the expression in the whole abdomen of the fly may mimic significant reductions in the expression of immune peptides in organs where the trypanosomes develop such as the midgut. Other factors that are induced by starvation and that may affect the development of a trypanosomal infection in tsetse can, however, not be excluded. They include midgut lectins, anti-oxidants and symbiotic associations in tsetse (Abubakar et al., 2006; Munks et al., 2005; Macleod et al., 2007; Roditi and Lehane, 2008).

Although these observations are based entirely on laboratory experiments, the outcome may contribute to a better understanding of the seasonal dynamics of trypanosomal infection rates in field populations of tsetse flies. Indeed, the monthly metacyclic infection rate of tsetse populations does undergo substantial variations over the year. Such variations have been attributed to changes in the survival rate of the tsetse population or the availability of infected hosts (Jordan, 1965; Harley, 1967). Our results suggest that seasonal stress situations, resulting in changes in the susceptibility to trypanosomal infections of emerging teneral flies, could also play a major role. Especially the hot dry season when ambient temperatures are high constitutes a stressful period for tsetse flies. This is reflected in the decrease in the fly's body size and fat body level during this season (Jackson, 1952; Glasgow and Bursell, 1960; Dransfield et al., 1989). The association between high ambient temperatures, stress in tsetse flies and the concomitant increase in the tsetse's susceptibility to trypanosomal infections may offer an explanation for the often observed relationship between high temperatures and high trypanosomal infection rates in tsetse. For example, Kinghorn and York, 1912 and Burtt (1946) reported higher susceptibility to infection with $T$. $b$. rhodesiense of flies emerging from pupae incubated at a high temperature. The same association may explain partly Fairbairn's (1948) observation that in the western G. morsitans belt of Tanzania the monthly mean maximum temperature and the number of diagnosed cases of sleeping sickness was significantly correlated. Finally and on a continental scale, a review of surveys determining the infection rates of savannah species of tsetse flies conducted over a period of 45 years also showed a positive correlation between infection rate of tsetse flies and the mean annual temperature (Ford and Leggate, 1961). Nevertheless, further research is required to determine the field implications of our laboratory observations.

\section{References}

Abubakar, L.U., Bulimo, W.D., Mulaa, F.J., Osir, E.O., 2006. Molecular characterization of a tsetse fly midgut proteolytic lectin that mediates differentiation of African trypanosomes. Insect Biochem. Mol. Biol. 36, 344-352.

Aksoy, S., Gibson, W., Lehane, M.J., 2003. Interactions between tsetse and trypanosomes with implications for the control of trypanosomiasis. Adv. Parasitol. $53,1-83$.

Attardo, G.M., Strickler-Dinglasan, P., Perkin, S.A.H., Caler, E., Bonaldo, M.F., Soares, M.B., El-Sayeed, N., Aksoy, S., 2006. Analysis of fat body transcriptome from the adult tsetse fly. Glossina morsitans morsitans. Insect Mol. Biol. 15, 411-424.

Boulanger, N., Brun, R., Ehret-Sabatier, L., Kunz, C., Bulet, P., 2002. Immunopeptides in the defense reactions of Glossina morsitans to bacterial and Trypanosoma bruce brucei infections. Insect Biochem. Mol. Biol. 32, 369-375.

Burtt, E.D., 1946. Incubation of tsetse pupae: increased transmission rate of Try panosoma rhodesiense in Glossina morsitans. Ann. Trop. Med. Parasitol. 40, 18-28.

Denlinger, D.L., Ma, W.C., 1974. Dynamics of the pregnancy cycle in the tsetse fly Glossina morsitans. J. Insect. Physiol. 20, 1015-1026.

Dransfield, R.D., Brightwell, R., Kiilu, J., Chaudhury, M.F.B., Adabie, D.A., 1989. Size and mortality rates of Glossina pallidipes in the semi-arid zone of southwestern Kenya. Med. Vet. Entomol. 3, 83-95.

Elsen, P., Van Hees, J., De Lil, E., 1993. L'historique et les conditions d'élevage des lignées de glossines (Diptera, Glossinidae) maintenues à l'Institut de Médecine Tropical Prince Léopold d'Anvers. J. Afr. Zool. 107, 439-449.

Fairbairn, H., 1948. Sleeping sickness in Tanganyika territory, 1922-1946. Trop. Dis. Bull. 45, 1-17.

Fairbairn, H., Culwick, A.T., 1950. The transmission of polymorphic trypanosomes. Acta Trop. 7, 19-47.

Fairbairn, H., Watson, H.J., 1955. The transmission of Trypanosoma vivax by Glossina palpalis. Ann. Trop. Med. Parasitol. 49, 250-259.

Ford, J., Leggate, B.M., 1961. The geographical and climatic distribution of trypanosome infection rates in Glossina morsitans group of tsetse-flies. Trans. $\mathrm{R}$. Soc. Trop. Med. Hyg. 55, 383-397.

Geigy, R., Kauffman, M., 1973. Sleeping sickness survey in the Serengeti area (Tanzania) 1971. I. Examination of large mammals for trypanosomes. Acta Trop. 30, $12-23$.

Glasgow, J.P., Bursell, E., 1960. Seasonal variations in the fat content and size of Glossina swynnertoni Austen. Bull. Entomol. Res. 51, 705-713.

Hao, Z., Kasumba, I., Lehane, M.J., Gibson, W.C., Kwon, J., Aksoy, S., 2001. Tsetse immune responses and trypanosome transmission: implications for the development of tsetse-based strategies to reduce trypanosomiasis. Proc. Natl. Acad. Sci. U.S.A. 98, 12648-12653.

Harley, J.M.B., 1967. Further studies on age and infection rate of Glossina pallidipes, G. palpalis fuscipes and G. brevipalpis in Uganda. Bull. Entomol. Res. 57, 459-477.

Jackson, C.H.N., 1952. Seasonal variations in the mean size of tsetse flies. Bull. Entomol. Res. 43, 703-706.

Jordan, A.M., 1965. The hosts of Glossina as the main factor affecting Trypanosome infection rates of tsetse flies in Nigeria. Trans. R. Soc. Trop. Med. Hyg. 59, 423-431.

Kinghorn, A., York, W., 1912. On the influence of metrological conditions on the development of Trypanosoma rhodesiense in Glossina morsitans. Ann. Trop. Med. Parasitol. 6, 405-413.

Koella, J.C., Sörensen, F.L., 2002. Effect of adult nutrition on the melanisation immune response of the malaria vector Anopheles stephensi. Med. Vet. Entomol. 16, 316-320.

Kubi, C., Van Den Abbeele, J., De Deken, R., Marcotty, T., Van den Bossche, P., 2006. The effect of starvation on the susceptibility of teneral and non-teneral tsetse flies to trypanosome infection. Med. Vet. Entomol. 20, 388-392.

Langley, P.A., Hargrove, J.W., Wall, R.L., 1990. Maturation of the tsetse fly Glossina pallidipes (Diptera: Glossinidae) in relation to trap-orientated behaviour. Physiol Entomol. 15, 179-186.

Leak, S.G.A., 1998. Tsetse Biology and Ecology: Their Role in the Epidemiology and Control of Trypanosomosis. CABI Publishing, Wallingord Oxon, p. 592.

Lehane, M.J., Aksoy, S., Levashina, E., 2004. Immune responses and parasite trans mission in blood-feeding insects. Trends Parasitol. 20, 433-439.

Le Ray, D., Barry, J.D., Easton, C., Vickerman, K., 1977. First tsetse fly transmission of the 'Antat' serodeme of Trypanosoma brucei. Ann. Soc. Belg. Med. Trop. 57, 369-381. 
Llyod, L., Johnson, W.B., 1924. The trypanosome infections of tsetse flies in northern Nigeria and a new method of estimation. Bull. Entomol. Res. 14, 265-288.

Macleod, E.T., Maudlin, I., Darby, A.C., Welburn, S.C., 2007. Antioxidants promote establishment of trypanosome infections in tsetse. Parasitology 134, 827-831.

Munks, R.J., Sant'Anna, M.R., Grail, W., Gibson, W., Igglesden, T., Yoshiyama, M., Lehane, S.M., Lehane, M.J., 2005. Antioxidant gene expression in the bloodfeeding fly Glossina morsitans morsitans. Insect Mol. Biol. 14, 483-491.

Ndegwa, P.N., Irungu, L.W., Moloo, S.K., 1992. Effect of puparia incubation temperature: increased infection rates of Trypanosoma congolense in Glossina morsitans centralis, G. fuscipes fuscipes and G. brevipalpis. Med. Vet. Entomol. 6, 127-130.

Phelps, R.J., Clarke, G.P.Y., 1974. Seasonal elimination of some size classes in male Glossina morsitans morsitans Westw. Bull. Entomol. Res. 64, 313-324.

Roditi, I., Lehane, M.J., 2008. Interactions between trypanosomes and tsetse flies. Curr. Opin. Microbiol. 11, 345-351.
Siva-Jothy, M., Thompson, J.J.W., 2002. Short-term nutrient deprivation affects immune function. Physiol. Entomol. 27, 206-212.

StataCorp, 2006. Stata Statistical Software: Release 9.2. Texas: Stata Corporation.

Taylor, A.W., 1932. The development of West African strains of Trypanosoma gambiense in Glossina tachinoides under normal laboratory conditions and at raised temperatures. Parasitology 24, 401-418.

Van den Bossche, P. Hargrove, J.W., 1999. Seasonal variation in nutritional levels of male tsetse flies Glossina morsitans morsitans Westwood (Diptera: Glossinidae) caught using fly-rounds and electric screens. Bull. Entomol. Res. 89, 381-387.

Vandesompele, J., De Preter, K., Pattyn, F., Poppe, B., Van Roy, N., De Paepe, A., Speleman, F., 2002. Accurate normalization of real-time quantitative RT-PCR data by geometric averaging of multiple internal control genes. Genome Biol., 3. 